\title{
VANTAGENS DA AMAMENTAÇÃO E ALTERAÇÕES NO ESTILO DE VIDA DA LACTANTE
}

\section{ADVANTAGES OF BREASTFEEDING AND CHANGES IN LIFESTYLE OF LACTATING}

\author{
Ernandes Gonçalves Dias, ${ }^{1}$ Ana Lúcia Santana Araújo Freitas, ${ }^{1,2}$ Héllen Christine Silva Cunha Martins, ${ }^{1}$ Kamila \\ Pinto Martins, ${ }^{1}$ Janine Cinara Silveira Alves ${ }^{1}$ \\ ${ }^{1}$ Faculdade Presidente Antônio Carlos/Porterinha/Brasil. ${ }^{2}$ Hospital e Maternidade Nossa Senhora das Graças/Monte Azul/Brasil. \\ Autor correspondente: Ernandes Gonçalves Dias \\ e-mail: ernandesgdias@yahoo.com.br
}

\section{EDITORES}

Thiago Gomes Heck

(Unijuí-Brasil)

Adriane Cristina Bernat Kolankiewicz

(Unijuí-Brasil)

\section{EDITORES DE ÁREA}

Educação \& Saúde

Eva Teresinha de Oliveira Boff

(Unijuí-Brasil)

Fisioterapia \& Saúde

Eliane Roseli Winkelmann

(Unijuí-Brasil)

Ciências Farmacêuticas \& Saúde

Marilei Uecker Pletsch

(Unijuí-Brasil)

Nutrição \& Saúde

Lígia Beatriz Bento Franz

(Unijuí-Brasil)

Nadia Oliveira

(Unipampa-Brasil)

Ingrid Perry

(UNESC-Brasil)

Enfermagem e suas contribuições para a prática

Adriane Cristina Kolankiewicz

(Unijuí-Brasil)

Crhis de Brum

(UFFS-Brasil)

Neila de Souza

(UFSM-Brasil)

Exercício Físico \& Saúde

Thiago Gomes Heck

(Unijuí-Brasil)

Anderson Zampier Ulbrich

(UFPR)

\section{Editora Unijuí}

Universidade Regional do Noroeste do

Estado do Rio Grande do Sul (Unijuí)

\section{RESUMO}

Este estudo teve como objetivo verificar o conhecimento sobre as vantagens da amamentação e as alterações ocorridas no estilo de vida da lactante em virtude da amamentação. Trata-se de um estudo descritivo e exploratório de natureza quanti-qualitativa, realizado com 12 lactantes na Unidade de Saúde Durval José de Oliveira. Os dados foram coletados no período de março a abril de 2014 por meio de uma entrevista aplicada na residência da lactante. Os resultados revelaram que $41,7 \% \pm 1,8$ tinham idade entre 26 e 30 anos, 83,3\% 55,7 casadas, $50 \% \pm 1,9$ do lar, $50 \% \pm 2$ tinham $2^{\circ}$ grau completo, $66,7 \% \pm 2,8$ tiveram gestações prévias, $75 \%$ não desmamaram seu bebê precocemente mesmo enfrentando alterações em seu estilo de vida e/ou amamentando de forma mista. As lactantes reconhecem a proteção contra doenças como vantagem do aleitamento materno tanto para a criança quanto para si. As alterações no estilo de vida foram mudanças no padrão de sono, adaptações no trabalho e afazeres domésticos para atender às necessidades da criança. Concluiu-se que as lactantes, apesar de terem conhecimentos limitados quanto às vantagens da amamentação e apresentarem alterações em seu estilo de vida, permitiram que essas ocorrências impedissem a prática da amamentação.

Palavras-chave: Conhecimento. Aleitamento materno. Estilo de vida. 


\section{Revista}

\section{Contexto}

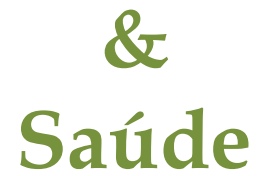

Volume 16

Número 31

2016

ISSN 2176-7114

\begin{abstract}
A Revista Contexto \& Saúde é um periódico do Departamento de Ciências da Vida da Universidade Regional do Noroeste do Estado do Rio Grande do Sul (Unijuí). É um periódico semestral que tem por objetivo a divulgação da produção técnico-científica de temas relacionados à área de Ciências da Saúde.
\end{abstract}

O escopo da revista abrange a divulgação de resultados de pesquisa que contemplem avanços no processo saúde-doençacuidado e no conhecimento e aplicabilidade de novos processos químicos e biológicos em saúde.

Neste periódico, entende-se que a publicação de estudos com os aspectos epidemiológicos, assistenciais e educacionais em saúde, experimentais e aplicados é uma forma a subsidiar e qualificar a atenção à saúde de modo interdisciplinar.

\begin{abstract}
This study aimed to verify the knowledge about the advantages of breastfeeding and changes in the lifestyle of breast-feeding function. It is a descriptive and exploratory study of quantitative and qualitative nature, accomplished with 12 lactating at the Health Unit Durval José of Oliveira. Data were collected from March to April 2014 by interviewing applied in the residence of lactating. The results revealed that $41.7 \% \pm 1.8$ were aged between 26 to 30 years, $83.3 \% \pm 5.7$ married, $50 \% \pm 1.9$ Housewife, $50 \% \pm 2$ had 2 School graduate, $66,7 \% \pm$ 2.8 had previous pregnancies, $75 \%$ did not weaned her baby precocious same facing changes in your lifestyle and / or breastfeeding in mixed manner. The lactating recognize the protection against diseases such as breastfeeding advantage both for the child and themselves. The changes in lifestyle have been changes in sleep patterns, adaptations in work and household chores to meet the child's needs. It was concluded the lactating even though they have limited knowledge about the advantages of breastfeeding and present changes in your lifestyle, these events did not stop the practice of breastfeeding.
\end{abstract}

Keywords: Knowledge. Breast Feeding. Life Style. 


\section{INTRODUÇÃO}

A amamentação exclusiva é fundamental para o crescimento e desenvolvimento da criança, bem como para proteção contra diversas doenças, além de não representar ônus para o orçamento familiar. A introdução de outros alimentos, além do leite materno, nos primeiros quatro meses de vida da criança, pode interferir negativamente na absorção dos nutrientes e levar à diminuição da ingestão do leite materno, menor peso ponderal e ao aumento do risco de diarreias, infecções e alergias (SILVA; PESSOA, 2012).

Segundo Silva et al. (2011), menos de 35\% das crianças do mundo são alimentadas exclusivamente ao seio pelos primeiros quatro meses de vida, e as práticas de alimentação complementar são frequentemente inconvenientes e perigosas.

Nesse sentido, a Organização Mundial de Saúde preconiza a amamentação exclusiva durante os primeiros seis meses de vida da criança, assim como a sua continuação de forma complementada até os dois anos. Além de ser econômico, o aleitamento materno auxilia no combate à desnutrição e à mortalidade infantil por enfermidades comuns da infância. Os benefícios desta prática são evidentes em termos de saúde da criança, desenvolvimento intelectual e motor, menor risco de doenças crônicas e saúde materna (BRASIL, 2009).

Muito embora se reconheça a tendência ascendente do aleitamento materno no Brasil, dados da II Pesquisa de Prevalência de Aleitamento Materno nas Capitais Brasileiras e Distrito Federal, realizada em 2008, mostram que somente $9,3 \%$ das crianças amamentam de forma exclusiva até o $6^{\circ}$ mês de vida (RAMOS et al., 2010).

Para Fraifer e Santos (2011), o aleitamento, além de ser fundamental para o bebê, traz benefícios também para a mãe. Durante a amamentação a puérpera pode perceber várias vantagens, como a diminuição da hemorragia pós-parto, rápida involução uterina e recuperação mais rápida do peso. Além disso, verifica-se também a melhoria da remineralização óssea pós-parto, menor risco de câncer de ovário e de mama e evita a anemia e o aparecimento precoce da ovulação em razão da amenorreia lactacional.

Amamentar envolve aspectos biológicos, psicológicos, culturais, econômicos e sociais da puérpera que, somados, podem intervir no sucesso ou não desta prática. Neste sentido, é importante destacar que as ações de promoção devam considerar o meio onde a mãe vive e os fatores que podem dificultar o aleitamento (COELHO; 2010; NICK, 2011).

Os fatores determinantes do desmame estão relacionados a causas fisiológicas e ao estilo de vida. Entre estes fatores destacam-se a pega incorreta, mamilos dolorosos e/ou fissuras, ingurgitamento mamário, mastite e aspectos socioculturais, como as experiências vivenciadas pela mulher, sua percepção acerca do leite materno e sobre si mesma enquanto nutriz, a idade materna, a baixa escolaridade e o fato de a mesma trabalhar, às vezes, fora do ambiente familiar, a responsabilidade com o bebê e na manutenção da relação conjugal, entre outros (BRASIL, 2012; SILVA; PESSOA, 2012).

As mulheres que têm mudanças em seu estilo de vida associam as alterações ao fato de quererem diminuir os riscos para o bebê e para si. A adoção de estilos de vida mais saudáveis está também relacionada às mulheres quererem estar disponíveis às demandas da criança (COUTINHO et al., 2014).

Neste contexto, o apoio dos profissionais de saúde é importante, pois estes lidam diretamente no auxílio e cuidado de mulheres e crianças no período de amamentação. Este apoio permite garantir a cada mãe uma escuta 
ativa, quando os profissionais vão ouvir, entender e esclarecer as crenças e tabus que podem facilitar o desmame precoce (NICK, 2011).

Desta forma, é fundamental a atuação do profissional de enfermagem no incentivo e apoio ao aleitamento materno, por isso devem estar preparados para promover uma assistência integral e humanizada à mulher, de forma que respeite o saber e a história de vida de cada sujeito, para que possam superar os seus medos, dificuldades e inseguranças (SILVA; PESSOA, 2012).

Diante destes fatos e das experiências vivenciadas nos estágios curriculares nas Unidades de Saúde do município de Porteirinha-MG, surgiu o interesse pelo tema ao perceber o quantitativo de lactantes e, nessa fase, a mulher encontra-se vulnerável a diversas situações que podem colaborar para o desmame precoce, como as alterações no estilo de vida ou até o desconhecimento das vantagens da amamentação.

Dessa forma, o estudo teve como objetivo verificar o conhecimento sobre as vantagens da amamentação e as alterações ocorridas no estilo de vida da lactante em razão da amamentação, entre as lactantes usuárias da Unidade Básica de Saúde Durval José Oliveira.

\section{METODOLOGIA}

Considerando o objetivo proposto, o estudo caracteriza-se como descritivo e exploratório de natureza quantiqualitativa, realizado na Unidade Básica de Saúde Durval José Oliveira, localizada no Bairro Vila Serranópolis, na cidade de Porteirinha, norte de Minas Gerais.

Os sujeitos da pesquisa foram as lactantes cadastradas na Unidade supracitada independente de cor, raça, religião ou classe social, com idade igual ou superior a 18 anos e que aceitaram participar do estudo pela assinatura do Termo de Consentimento Livre e Esclarecido, conforme determina a Resolução 466/2012.

Para a obtenção dos dados foi utilizada uma entrevista estruturada, gravada em áudio, aplicada a todas as lactantes (12 no momento da coleta) em seu domicílio, em data e horário previamente agendados, no período de março a abril de 2014. Ressalta-se que, antes de iniciar a coleta dos dados, foi realizada, a partir dos cadastros de acompanhamento mensal de gestantes, a busca das lactantes com os seus respectivos endereços para o agendamento da entrevista.

Os dados quantitativos foram processados em um microcomputador mediante uma planilha do Microsoft Excel 2010. Após análise e interpretação desses dados foram construídas tabelas com a finalidade de facilitar a compreensão dos dados levantados, considerando que, acompanhado à exposição desses elementos gráficos, foi realizada uma discussão confrontando o referencial teórico com o dado coletado.

Em relação à análise qualitativa foram realizadas leituras sucessivas dos depoimentos. Estes foram agrupados até que se obtivesse saturação das informações e, na sequência, foi realizada a interpretação das mesmas e a comparação com a literatura específica.

O projeto deste estudo foi analisado pelo Comitê de Ética e Pesquisa da Universidade Presidente Antônio Carlos e aprovado com o parecer número 645.191.

\section{RESULTADOS E DISCUSSÃO}


A Tabela 1 mostra que, entre as entrevistadas, $5(41,7 \%)$ lactantes tinham idade entre 26 e 30 anos, $4(33,3 \%)$ entre 31 e 35 anos, $2(16,7 \%)$ entre 36 e 40 anos e $1(8,3 \%)$ entre 20 e 25 anos de idade $( \pm 1,8)$.

Estes achados corroboram os dados encontrados por Ciampo et al. (2008), em uma pesquisa realizada com mães de crianças com idade de até seis meses completos, cadastradas em programas de puericultura das Unidades Básicas de Saúde de Ribeirão Preto-SP no ano de 2006; a maioria das lactantes tinha idade entre 20 e 29 anos $(57,1 \%)$.

De acordo com Aragaki e Silva (2011), a idade materna é um dado importante para avaliar a mulher em seu ciclo gravídico-puerperal, e possui capacidade de interferir na saúde da mãe e do bebê desde a gestação até o pós-parto.

Com relação ao estado civil, os dados revelam que $10(83,3 \%)$ lactantes eram casadas e $2(16,7 \%)$ solteiras $( \pm 5,7)$. Nota-se, neste estudo, prevalência de mulheres casadas. Segundo Batista, Farias e Melo (2013), a presença do companheiro pode favorecer a amamentação, principalmente se estimular, apoiar e ajudar nas tarefas em geral, tanto da casa quanto nos cuidados com o bebê.

Quanto à ocupação das lactantes, os dados mostram que $6(50 \%)$ eram do lar, $1(8,3 \%)$ assistente administrativo, $1(8,3 \%)$ técnica em higiene bucal, $1(8,3 \%)$ lavadeira, $1(8,3 \%)$ cabeleireira, $1(8,3 \%)$ professora e $1(8,3 \%)$ auxiliar de secretaria.

Tabela 1 - Caracterização das lactantes da Unidade de Saúde Durval José Oliveira. Porteirinha-MG, 2014.

\begin{tabular}{|c|c|c|c|}
\hline Variáveis & $\mathbf{N}^{\circ}$ & $\%$ & $\pm(\mathbf{N})$ \\
\hline \multicolumn{4}{|c|}{ Idade } \\
\hline $20-26$ & 1 & 8,3 & \\
\hline $26-31$ & 5 & 41,7 & \\
\hline $31-36$ & 4 & 33,3 & \\
\hline $36 \mathrm{H} 40$ & 2 & 16,7 & 1,8 \\
\hline \multicolumn{4}{|c|}{ Estado civil } \\
\hline Casada & 10 & 83,3 & \\
\hline Solteira & 2 & 16,7 & 5,7 \\
\hline \multicolumn{4}{|c|}{ Ocupação } \\
\hline Do lar & 6 & 50 & \\
\hline Assistente administrativo & 1 & 8,3 & \\
\hline Cabeleireira & 1 & 8,3 & \\
\hline Técnica de higiene bucal & 1 & 8,3 & \\
\hline Lavadeira & 1 & 8,3 & \\
\hline Professora & 1 & 8,3 & \\
\hline Auxiliar de secretaria & 1 & 8,3 & 1,9 \\
\hline \multicolumn{4}{|c|}{ Escolaridade } \\
\hline $1^{\circ} \mathrm{Grau}$ completo & 2 & 16,7 & \\
\hline $2^{\circ} \mathrm{Grau}$ completo & 6 & 50 & \\
\hline Superior completo & 4 & 33,3 & 2 \\
\hline \multicolumn{4}{|l|}{ Gestações Anteriores } \\
\hline Não & 4 & 33,3 & \\
\hline Sim & 8 & 66,7 & 2,8 \\
\hline
\end{tabular}

Fonte: Dados da Pesquisa. 2014. 
A prevalência de lactantes do lar também foi observada em uma pesquisa realizada por Vitor et al. (2010) em Unidade de Saúde, Creches e Hospitais de duas regiões do Rio Grande do Sul com mães lactantes, quando constataram que $62,9 \%$ das lactantes eram também donas de casa.

Quanto à escolaridade, $6(50 \%)$ tinham $2^{\circ}$ grau completo, $4(33,3 \%)$ o curso superior completo e $2(16,7 \%)$ o $1^{\circ}$ grau completo $( \pm 2)$. Estes dados revelam que a população estudada tem um nível de escolaridade considerado adequado para a compreensão de informações sobre a amamentação.

Os dados corroboram com os encontrados por Fragoso e Fortes (2011) em uma pesquisa realizada na maternidade do Hospital Regional da Asa Sul do Distrito Federal, que verificaram que 52,9\% da amostra também tinham escolaridade maior, uma vez que 44,2\% concluíram o segundo grau e 8,8\% possuíam nível superior completo.

De acordo com o autor supracitado, a escolaridade da mãe facilita o aprendizado durante o pré-natal, interferindo na duração da amamentação. A escolaridade materna está associada a sua inserção no mercado de trabalho, dessa forma pode interferir negativamente sobre o aleitamento exclusivo.

Conforme Oliveira e Marques (2011), as lactantes com menos anos de estudo têm maior dificuldade de compreender as informações sobre o aleitamento; já as que possuem mais anos de estudo absorvem mais informações.

No que diz respeito ao número de gestações anteriores, observa-se que $8(66,7 \%)$ lactantes tiveram gestações prévias e $4(33,3 \%)$ não tiveram gestações anteriores $( \pm 2,8)$. Nota-se que a maioria das entrevistadas eram multíparas (66,7\%), o que vai contra os dados encontrados por Salvador et al. (2012) em uma pesquisa realizada no ambulatório de consulta de enfermagem pós-natal do Hospital Barão de Lucena, que verificaram que 53,4\% das entrevistadas eram primíparas e 46,6\% eram multíparas.

No que se refere aos conhecimentos sobre as vantagens da amamentação, todas as lactantes relataram conhecer, porém frisam em seus discursos a proteção contra as doenças como vantagem do aleitamento materno tanto para a criança quanto para a mãe, conforme mostra os seguintes relatos:

"[...] têm os nutrientes necessários para o bebê, são nutrientes, vitaminas e tudo que o bebê necessita e até o $6^{\circ}$ mês não necessita de água porque o leite já tem água e todas as vitaminas que necessita” (J. C. A. S., 35 anos).

"[...] porque o bebê evita ter doenças, né? Não vai ter infecções e aumenta a imunidade da criança” (A. A. C., 26 anos).

"Porque é saudável pra ele, né? E até mesmo pra gente mesmo. E eu já ouvi falar também que amamentar previne câncer de mama, não sei se é verdade” (G. C. C., 27 anos).

“[...] o leite materno evita muita coisa como doenças e é saudável, né?” (A. A. F., 33 anos).

Para Martins e Santana (2013), o leite materno traz vantagens imunológicas, psicológicas e nutricionais ao bebê, sendo também fundamental para a saúde materna. Em um estudo realizado por Azevedo et al. (2010) envolvendo puérperas do Alojamento Conjunto da Maternidade Escola Assis Chateaubriand em Fortaleza-CE, 
observou-se que $14,7 \%$ das lactantes reconheceram a importância nutricional como benefício para o bebê e $48 \%$ a importância imunológica.

Conforme Martins e Santana (2013), os conhecimentos das lactantes em relação à amamentação contribuem para que tenha sucesso durante a prática, mas não determinam que a amamentação seja realizada com eficácia.

Para Grando e Zuse (2011), a amamentação não é apenas nutrir a criança, mas também um processo de vínculo entre mãe e filho, capaz de repercutir no estado nutricional do bebê, proporcionando benefícios para ambos.

Diogo, Souza e Zocche (2011) asseveram que não basta a lactante ter conhecimento das vantagens da amamentação e querer realizar essa prática; é preciso que a mesma esteja integrada em um meio favorável à amamentação e conte com o apoio de profissionais capacitados.

A amamentação provoca alterações no estilo de vida da lactante para atender às necessidades do bebê. Possivelmente as alterações necessárias podem facilitar o desmame precoce ou amamentação mista anteriormente ao sexto mês de vida da criança. Nos discursos das lactantes observou-se relatos de alterações no padrão de sono, adaptações no trabalho fora de casa e nos afazeres domésticos, como alterações no estilo de vida que dificultam a amamentação, conforme os discursos a seguir:

“Acordo várias vezes à noite, e fico com sono o dia todo” (E. A. R. A., 33 anos).

“Eu trabalho o dia todo e tenho que vim correndo dá peito” (A. A. F., 33 anos).

“Depois que meu filho nasceu, é eu não dô conta mais de fazer os serviços de casa” (A. A. C., 26 anos).

"Para mim é difícil, tenho que trabalhar cedo e o bebê mama muito à noite. Fico com sono no trabalho, atrapaia” (G. C. C., 27 anos).

Em uma pesquisa realizada por Shimoda e Silva (2010) no Centro de Saúde da Escola Professor Samuel Barnsley Pessoa, no município de São Paulo, com 238 lactantes, verificou-se que 72,1\% das lactantes referiram sofrer alteração no padrão de sono. O repouso da lactante está vinculado às demandas das mamadas e ao sono do bebê; esse fator está presente desde o nascimento da criança.

Shimoda et al. (2013) afirmam que durante a prática do aleitamento, especialmente o exclusivo, as lactantes sofrem alterações no padrão de sono em razão das demandas das mamadas noturnas.

Autores como Rocha et al. (2010) e Garcia et al. (2013) destacam, em seus estudos, que o estilo de vida moderno, com a inserção da mulher no mercado de trabalho, pode influenciar diretamente na prática da amamentação. Está condição, porém, não deve ser decisiva na atitude de amamentar, pois as empresas já vêm se manifestando favoravelmente neste sentido.

Apesar das alterações no estilo de vida, entre as lactantes estudadas 9 (75\%) conseguiram amamentar mesmo que de forma não exclusiva. As $3(25 \%)$ que desmamaram precocemente foi por causa da fissura mamária (2; $66,7 \%)$ ou ingurgitamento $(1 ; 33,3 \%)$.

No estudo de Castro et al. (2009), realizado em lactantes da Maternidade Pública do município de João Pessoa$\mathrm{PB}$, verificou-se que 28,3\% das lactantes apresentavam ingurgitamento mamário e 7,6\% fissura mamilar. Para 
os autores, as intercorrências mamárias relacionadas à lactação, entre elas o ingurgitamento, as físsuras e a mastite, são reconhecidas como fatores determinantes no insucesso da amamentação.

\section{CONSIDERAÇÕES FINAIS}

O estudo revela que as lactantes têm conhecimento sobre as vantagens da amamentação mesmo que de forma limitada. As principais mudanças no estilo de vida foram alteração no padrão de sono, adaptação no trabalho e afazeres domésticos. As alterações no estilo de vida, no entanto, não causaram desmame precoce, pois este esteve associado a causas orgânicas e fisiológicas.

A decisão de amamentar, mesmo com os obstáculos do estilo de vida, pode ser reflexo de um acompanhamento pré-natal realizado de forma satisfatória, quando, enquanto gestantes, compreenderam a necessidade de amamentar sua criança. As vantagens da amamentação devem ser continuamente reforçadas nas ações individuais e coletivas para que todas as gestantes tenham acesso a essas informações.

\section{REFERÊNCIAS}

ARAGAKI, I. M. M.; SILVA, I. A. Nursing mothers' perception about their quality of life. Rev Esc Enferm USP, v. 45, n. 1, p. 69-76, 2011. Disponível em: <http://dx.doi.org/10.1590/S0080-62342011000100010>. Acesso em: maio 2014.

AZEVEDO, D. S. de et al. Conhecimento de primíparas sobre os benefícios do aleitamento materno. Rev. Rene. Fortaleza, v. 11, n. 2, p. 53-62, abr./jun. 2010.

BATISTA, K. R. de A.; FARIAS, M. do C. A. D. de; MELO, W. dos S. N. de M. Influência da assistência de enfermagem na prática da amamentação no puerpério imediato. Saúde em Debate, Rio de Janeiro, v. 37, n. 96, p. 130-138, jan./mar. 2013.

BRASIL. Ministério da Saúde. Secretaria de Atenção à Saúde. Departamento de Atenção Básica. Saúde da criança: nutrição infantil: aleitamento materno e alimentação complementar. Cadernos de Atenção Básica, Brasília: Editora do Ministério da Saúde, n. 23, 112p., 2009.

. Ministério da Saúde. Secretaria de Atenção à Saúde. Departamento de Atenção Básica. Aleitamento materno, distribuição de fórmulas infantis em estabelecimentos de saúde e a legislação. Brasília: Ministério da Saúde, 2012. 26 p.

CASTRO, K. F. de et al. Intercorrências mamárias relacionadas à lactação: estudo envolvendo puérperas de uma maternidade pública de João Pessoa, PB. O Mundo da Saúde, São Paulo, v. 33, n. 4, p. 433-439, 2009.

CIAMPO, L. A. D. et al. Aleitamento materno exclusivo: do discurso à prática. Pediatria, São Paulo, v. 30, n. 1, p. 22-26, 2008.

COELHO, C. V. A. Fatores que interferem e dificultam na duração do aleitamento materno: revisão da literatura. 2010. 31f. Trabalho (Monografia de Especialização em Atenção Básica em Saúde da Família) - Universidade Federal de Minas Gerais, Faculdade de Medicina, Núcleo de Educação em Saúde Coletiva, Governador Valadares, 2010.

COUTINHO, E. de C. et al. Gravidez e parto: O que muda no estilo de vida das mulheres que se tornam mães? Rev Esc Enferm USP, v. 48 (Esp2), p. 17-24, 2014. Disponível em: 〈http://dx.doi.org/10.1590/S0080-623420140000800004〉. Acesso em: ago. 2014.

DIOGO, E. F.; SOUZA, T.; ZOCCHE, D. de A. Causas do desmame precoce e suas interfaces com a condição socioeconômica e escolaridade. Enfermagem em Foco, v. 2, n. 1, p. 10-13, 2011.

FRAGOSO, A. P. R.; FORTES, R. C. Fatores associados à prática do aleitamento materno entre nutrizes de um hospital público do Distrito Federal. J Health Sci Inst., v. 29, n. 2, p. 114-8, 2011. 
FRAIFER, V. S. S.; SANTOS, E. E. A importância da exclusividade do aleitamento materno nos primeiros seis meses de vida. Diálogos \& Ciência, ano 9, n. 26, jun. 2011.

GARCIA, M. M. R. et al. O conhecimento das puérperas sobre preparo das mamas e aleitamento materno. Revista Eletrônica Gestão \& Saúde, v. 4, n. 1, p. 1.684-1.698, 2013.

GRANDO, T.; ZUSE, C. L. Amamentação exclusiva: um ato de amor, afeto e tranquilidade-relato de experiência. Vivências: Revista Eletrônica de Extensão da URI, v. 7, n. 13, p. 22-28, out. 2011.

MARQUES, R. de F. da S. V. et al. Fatores relacionados às dificuldades no aleitamento materno entre mães adolescentes da fundação santa casa de misericórdia do Pará. Revista Paraense de Medicina, v. 22, n. 1, jan./mar. 2008.

MARTINS, M. Z. O.; SANTANA, L. Benefícios da amamentação para saúde materna. Interfaces Científicas-Saúde e Ambiente, Aracaju, v. 1, n. 3, p. 87-97, jun. 2013. Disponível em: 〈http://dx.doi.org/10.17564/2316-3798.2013v1n3p87-97〉. Acesso em: abr. 2014.

NICK, M. S. A importância do aleitamento materno exclusivo nos primeiros seis meses de vida para a promoção da saúde da criança. 2011. 30f. Trabalho (Monografia em Especialização em Atenção Básica em Saúde da Família) - Universidade Federal de Minas Gerais, Faculdade de Medicina Teófilo Otoni, 2011.

OLIVEIRA, K. M. P. de; MARQUES, I. R. Situação do aleitamento materno no Brasil: uma revisão. Rev Enferm Unisa, v. 12, n. 1, p. 73-8, 2011.

RAMOS, C. V. et al. Prevalência do aleitamento materno exclusivo e os fatores a ele associados em crianças nascidas nos Hospitais Amigos da Criança de Teresina - Piauí. Epidemiol. Serv. Saúde, Brasília, v. 19, n. 2, p. 115-124, abr./jun. 2010.

ROCHA, N. B. et al. O ato de amamentar: um estudo qualitativo. Physis, Rio de Janeiro, v. 20, n. 4, dez. 2010. Disponível em: <http://dx.doi.org/10.1590/S0103-73312010000400012>. Acesso em: jul. 2014.

SALVADOR, J. P. et al. Participação do companheiro na promoção do aleitamento materno exclusivo em hospital amigo da criança. Rev. Interdisciplinar NOVAFAPI, Teresina. v. 5, n.1, p. 30-36, jan./fev./mar. 2012.

SHIMODA, G. T. et al. A. Health needs and quality of life of nursing mothers. Acta Paul Enferm., v. 26, n. 3, p. $213-218,2013$. Disponível em: <http://dx.doi.org/10.1590/S0103-21002013000300002>. Acesso em: jun. 2014.

SHIMODA, G. T.; SILVA, I. A. Necessidade de saúde de mulheres em processo de amamentação. Rev Bras Enferm, Brasília, v. 63, n. 1, p. 58-65, 2010, jan./fev. Disponível em: <http://dx.doi.org/10.1590/S0034-71672010000100010>. Acesso em: jul. 2014.

SILVA, M. D. B. et al. Suporte social na amamentação do prematuro egresso da unidade de terapia intensiva neonatal. Revista de Enfermagem UFPE on-line, v. 5, n. 10, p. 1.370-1.379, 2011. Disponível em: <http://dx.doi.org/10.5205/01012007>. Acesso em: ago. 2014.

SILVA, V. F. da; PESSOA, C. G. de O. Fatores determinantes do aleitamento materno exclusivo em uma cidade de Minas Gerais. Revista Enfermagem Integrada, Ipatinga, v. 5, n. 1, p. 867-879, 2012.

VITOR, R. S. et al. Aleitamento materno exclusivo: análise desta prática na região Sul do Brasil. Rev. da AMRIGS, Porto Alegre. v. 54, n. 1, p. 44-48, jan./mar. 2010. 